\title{
Size Effect in the Spin Glass Magnetization of Thin AuFe Films as Studied by Polarized Neutron Reflectometry
}

\author{
M. Saoudi and H. Fritzsche* \\ National Research Council Canada, Canadian Neutron Beam Centre, Chalk River Laboratories, \\ Bldg. 459, Chalk River, ON, KOJ 1J0, Canada \\ G. J. Nieuwenhuys \\ Research Department Condensed Matter Research with Neutrons and Muons, Paul-Scherrer Institute-Villigen, Switzerland \\ and Kamerlingh Onnes Laboratory, Leiden University-Leiden, The Netherlands \\ M. B. S. Hesselberth \\ Kamerlingh Onnes Laboratory, Leiden University-Leiden, The Netherlands
}

(Received 10 October 2007; published 6 February 2008)

\begin{abstract}
We used polarized neutron reflectometry to determine the temperature dependence of the magnetization of thin AuFe films with $3 \%$ Fe concentration. We performed the measurements in a large magnetic field of $6 \mathrm{~T}$ in a temperature range from 295 to $2 \mathrm{~K}$. For the films in the thickness range from 500 to $20 \mathrm{~nm}$ we observed a Brillouin-type behavior from $295 \mathrm{~K}$ down to $50 \mathrm{~K}$ and a constant magnetization of about $0.9 \mu_{B}$ per Fe atom below $30 \mathrm{~K}$. However, for the $10 \mathrm{~nm}$ thick film we observed a Brillouin-type behavior down to $20 \mathrm{~K}$ and a constant magnetization of about $1.3 \mu_{B}$ per $\mathrm{Fe}$ atom below $20 \mathrm{~K}$. These experiments are the first to show a finite-size effect in the magnetization of single spin-glass films in large magnetic fields. Furthermore, the ability to measure the deviation from the paramagnetic behavior enables us to prove the existence of the spin-glass state where other methods relying on a cusp-type behavior fail.
\end{abstract}

DOI: 10.1103/PhysRevLett.100.057204

PACS numbers: 75.50.Lk, 61.05.fj, 75.70.-i

During the last decades there has been a continuous theoretical and experimental interest in studying properties of spin-glass systems [1]. The prerequisite for a spin glass is a competition among the different interactions between the spins with a random distribution of these interactions. The challenge to develop concepts which describe disordered systems has been the main motivation for theorists in this field. For experimentalists the driving force has been to discover new magnetic properties, different from the well-understood paramagnets, antiferromagnets, and ferromagnets.

The random spin arrangement in a spin glass is frozen when cooling the system below a certain temperature $T_{f}$, called the freezing temperature. This temperature has been most often determined by measuring the magnetic susceptibility as a function of temperature and identifying the cusp as $T_{f}$ as it was done for the first time by Cannella and Mydosh [2]. Other measurements like the anomalous Hall effect [3] and Mössbauer effect [4] were successfully used to study the spin-glass phase transition, whereas other methods like resistivity [5-7] or magnetoresistance measurements [8] did not show any sharp features associated with a phase transition.

In recent years the focus has been on finite-size scaling, the crossover from two- to three-dimensional behavior as the film thickness is varied [9-11], and the existence of a surface anisotropy in thin spin-glass films [12-16]. It is very important to provide experimental data on the magnetization to prove or disprove certain models or theories.
However, from an experimental point of view it is extremely difficult to study finite-size effects in spin-glass systems because the magnetic signal in these diluted systems is tiny. Typically, the spin glass consists of only a few percent of magnetic atoms in a nonmagnetic host matrix. SQUID magnetometry is not adequate to measure the magnetization of spin-glass films in the nanometer range because of the small magnetic signal compared to the huge diamagnetic background of the substrate. We used SQUID data [17] to confirm that our $500 \mathrm{~nm}$ thick AuFe film exhibits the same magnetic properties as a bulk sample. Magnetometry experiments on thin spin-glass films reported in literature were all performed on multilayers in order to increase the signal $[10,11]$. However, it is always questionable whether multilayer data represent the true single film properties because of structural differences as the film gets thicker and an uncomplete decoupling of the individual spin-glass layers.

In this Letter we report on experiments using polarized neutron reflectometry (PNR) which has turned out only recently to be an excellent tool to determine the absolute magnetic moment in single thin spin-glass films $[18,19]$. We present for the first time a systematic study of the magnetic moment in thin spin-glass films in the thickness range from 500 to $10 \mathrm{~nm}$ during a field-cooling from 295 to $2 \mathrm{~K}$ in a field of $6 \mathrm{~T}$. We chose to cool the samples down in a large magnetic field to avoid time effects of the magnetization which occur when measuring the magnetization after a zero-field cooling [20,21]. These time effects are 
similar to the decay of the remanent magnetization in time and it is nowadays widely accepted that the field-cooled magnetization (rather than the zero-field cooled) represents the equilibrium magnetization of a spin glass [20-22]. This point of view is also supported by measurements of the magnetocaloric effect in CuMn [23].

A set of AuFe alloy films with an Fe concentration of 3 at. $\%$ and different thicknesses $t=10,20,50$ and $500 \mathrm{~nm}$ was prepared by co-sputtering high purity $\mathrm{Au}$ and Fe onto a silicon substrate. The films were analyzed using Rutherford backscattering and electron microprobe analysis showing that the thickness and composition were homogeneous. PNR experiments were performed on the C5 spectrometer at the neutron research reactor NRU in Chalk River, Canada. We used a $\mathrm{Cu}_{2} \mathrm{MnAl}$ Heusler crystal at $\lambda=$ $0.237 \mathrm{~nm}$ as monochromator and analyzer along with a PG filter [24] to reduce the higher order contamination $(\lambda / 2$ and $\lambda / 3$ ). The magnetic field was provided by a cryomagnet with the magnetic field being in the sample plane and perpendicular to the scattering plane. In this setup [25] we achieved a $96 \%$ polarization of the incoming neutron beam, as determined from the measured flipping ratio of 26 . The geometry of the experiment is the symmetrical $\theta-$ $2 \theta$ Bragg geometry in which the reflected angle $\theta_{f}$ is always equal to the incident angle $\theta_{i}\left(\theta=\theta_{i}=\theta_{f}\right)$. In this case the scattering vector $q$ is perpendicular to the film surface and is defined as $q=4 \pi \sin (\theta) / \lambda$.

Figure 1 displays the polarized neutron reflectivities $R^{+}$ (+) and $R^{-}$(open circles) as a function of the scattering vector $q$, measured at $10 \mathrm{~K}$ in a magnetic field of $6 \mathrm{~T}$ for the $20 \mathrm{~nm}$ thick $\mathrm{Au}_{97} \mathrm{Fe}_{3}$ film. $R^{+}$denotes the reflectivity for spin-up neutrons with spin parallel to the external magnetic field and $R^{-}$denotes the reflectivity for spin-down neu-

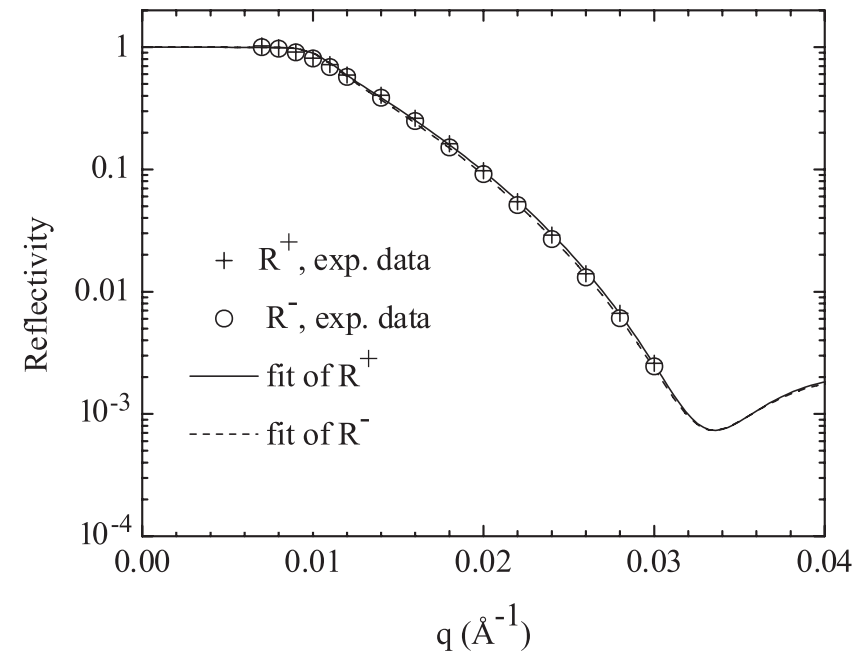

FIG. 1. Measured neutron reflectivities $R^{+}(+)$and $R^{-}$(open circles) as a function of the scattering vector $q$ for a $20 \mathrm{~nm}$ thick $\mathrm{Au}_{97} \mathrm{Fe}_{3}$ film along with the fits (solid and dashed line, respectively) at a temperature $T=10 \mathrm{~K}$ in an external magnetic field of $6 \mathrm{~T}$. trons with spin antiparallel to the external magnetic field. The reflectivity can be described with the Fermi pseudopotential (nuclear interaction) and a Zeeman term (magnetic interaction) [26-28]. For the spin-up neutrons the magnetic potential adds to the nuclear one, whereas for the spin-down neutrons the magnetic potential is subtracted from the nuclear potential. This leads to different reflectivity curves for the two spin states what is used to determine the magnetic moment of magnetic films. In our case $R^{+}$and $R^{-}$are nearly identical because the magnetization of the AuFe film is very small. Therefore, it is more convenient to plot the asymmetry $A$ defined as

$$
A=\left(R^{+}-R^{-}\right) /\left(R^{+}+R^{-}\right)
$$

as a function of $q$ in order to visualize the magnetic properties of the film.

Figure 2 shows the asymmetry for the $20 \mathrm{~nm}$ thick $\mathrm{Au}_{97} \mathrm{Fe}_{3}$ film plotted for $10 \mathrm{~K}$ (solid circles) and $200 \mathrm{~K}$ (open circles). The solid and dashed lines are the fit for the 10 and $200 \mathrm{~K}$ data, respectively. The solid line in Fig. 2 was obtained from the fits shown in Fig. 1 of $R^{+}$(solid line) and $R^{-}$(dashed line), using Eqn. (1). These fits were obtained by fitting simultaneously the spin-up and spin-down reflectivity using Parratt's recursion formalism [29]. We used a two-layer model that comprises of a single homogeneous AuFe film on a native silicon oxide layer (10 A thick) on top of a silicon substrate. For the $\mathrm{Si}$ and the $\mathrm{SiO}_{2}$ we used the scattering length densities (SLDs) of $2.073 \times 10^{-6}$ and $3.475 \times 10^{-6} \AA^{-2}$, respectively [30]. The only parameters which were fitted are the thickness, roughness, nuclear and magnetic SLD of the AuFe layer. The result for the $20 \mathrm{~nm}$ thick $\mathrm{Au}_{97} \mathrm{Fe}_{3}$ film at $10 \mathrm{~K}$ is a magnetic SLD of $0.044 \times$ $10^{-6} \AA^{-2}$ and a nuclear SLD of $4.31 \times 10^{-6} \AA^{-2}$. The

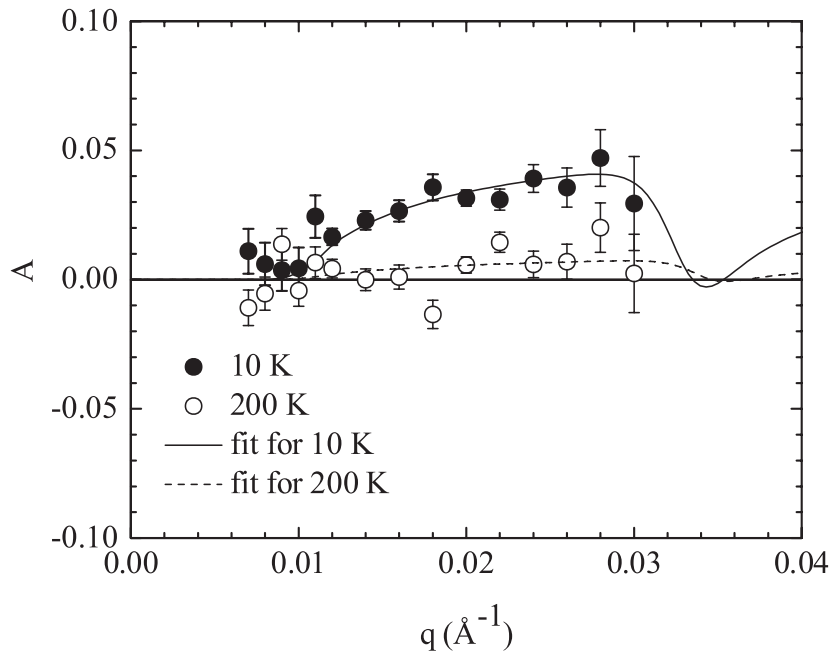

FIG. 2. Asymmetry as a function of the scattering vector $q$ for a $20 \mathrm{~nm}$ thick $\mathrm{Au}_{97} \mathrm{Fe}_{3}$ film measured at $10 \mathrm{~K}$ (solid circles) and measured at $200 \mathrm{~K}$ (open circles) in a magnetic field of $6 \mathrm{~T}$. The solid and dashed lines are the fits for the 10 and $200 \mathrm{~K}$ data, respectively. 
magnetic SLD was converted into an average magnetic moment $\bar{\mu}$ along the magnetic field of $0.9 \mu_{B}$ per $\mathrm{Fe}$ atom by assuming the same number density for the Fe as for the Au atoms. At $200 \mathrm{~K}$, the asymmetry is much smaller than at $10 \mathrm{~K}$ as can be seen in Fig. 2. The fit results in a magnetic SLD of $0.009 \times 10^{-6} \AA^{-2}$ which corresponds to a magnetic moment of $0.18 \mu_{B}$ per Fe atom.

The $500 \mathrm{~nm}$ thick film served as the bulk reference. The critical edge is determined by the SLD of the AuFe layer independent of the Si substrate. So, we simply used a AuFe substrate as the fit model. The magnetization calculated from our PNR measurements at $4 \mathrm{~K}$ is the same within errors as deduced from SQUID measurements on the identical sample [17]. These results agree well with magnetization measurements of bulk AuFe samples with an $\mathrm{Fe}$ concentration of 3.1 at.\% [31].

In a dilute AuFe system, above the spin-glass temperature, the Fe impurities do not interact and should behave as noninteracting paramagnetic moments. Along the applied field, the average magnetic moment per Fe impurity can be calculated by multiplying the magnetic moment $\mu$ of the impurity by the Brillouin function $\mathcal{B}(J, x)$ where $J$ is the total angular momentum and $x=\mu B / k_{B} T$ with $B$ the applied magnetic field, $k_{B}$ the Boltzmann constant, and $T$ the temperature. The magnetic moment $\mu$ of the impurity is $g \mu_{B} J$ with $g$ representing the Landé factor. The temperature dependence of the magnetization of $\mathrm{Au}_{97} \mathrm{Fe}_{3}$ films is shown in Fig. 3 for different thicknesses. The solid circles correspond to the values for the average magnetic moment $\bar{\mu}$ as derived from fits to the reflectivity curves. For comparison, the temperature dependence of the average magnetic moment at $6 \mathrm{~T}$ has been calculated taking $g=2$ and $J=2$ (solid line in Fig. 3). These values correspond to $4 \mu_{B}$ per $\mathrm{Fe}$ atom for noninteracting isolated $\mathrm{Fe}$ atoms. The Brillouin function describes the thin film data from $295 \mathrm{~K}$ down to about $T_{0}=50 \mathrm{~K}$ reasonably well for $t=20,50,500 \mathrm{~nm}$ [see Figs. 3(b) $-3(\mathrm{~d})]$. For $t=10 \mathrm{~nm}$ the Brillouin function describes the data down to about $T_{0}=20 \mathrm{~K}$ [see Fig. 3(a)]. The averaged magnetic moment at $10 \mathrm{~K}$ is $\bar{\mu}=1.3 \mu_{B}$ per Fe atom, whereas for $t \geq 20 \mathrm{~nm}$ the value is $0.9 \mu_{B}$ per Fe atom.

Below $T_{0}$, the impurity magnetization is reduced compared to the paramagnetic value. This reduction is due to the frustration in the spin-glass state. The observed temperature dependence of the magnetization can be described with a Brillouin-type function for isolated $\mathrm{Fe}$ atoms from room temperature down to a temperature $T_{0}$. That shows that $\mathrm{Fe}$ atoms in majority are dispersed homogeneously into the Au matrix as isolated atoms rather than clusters as found in $\mathrm{Au}_{93} \mathrm{Fe}_{7}$ film [18,19]. The deviation from the paramagnetic behavior at $T_{0}$ clearly proves the phase transition into a frustrated spin-glass system in a large magnetic field of $6 \mathrm{~T}$. In contrast, other methods like the anomalous Hall effect [3] and susceptibility measurements [2] were only able to determine a phase transition in low

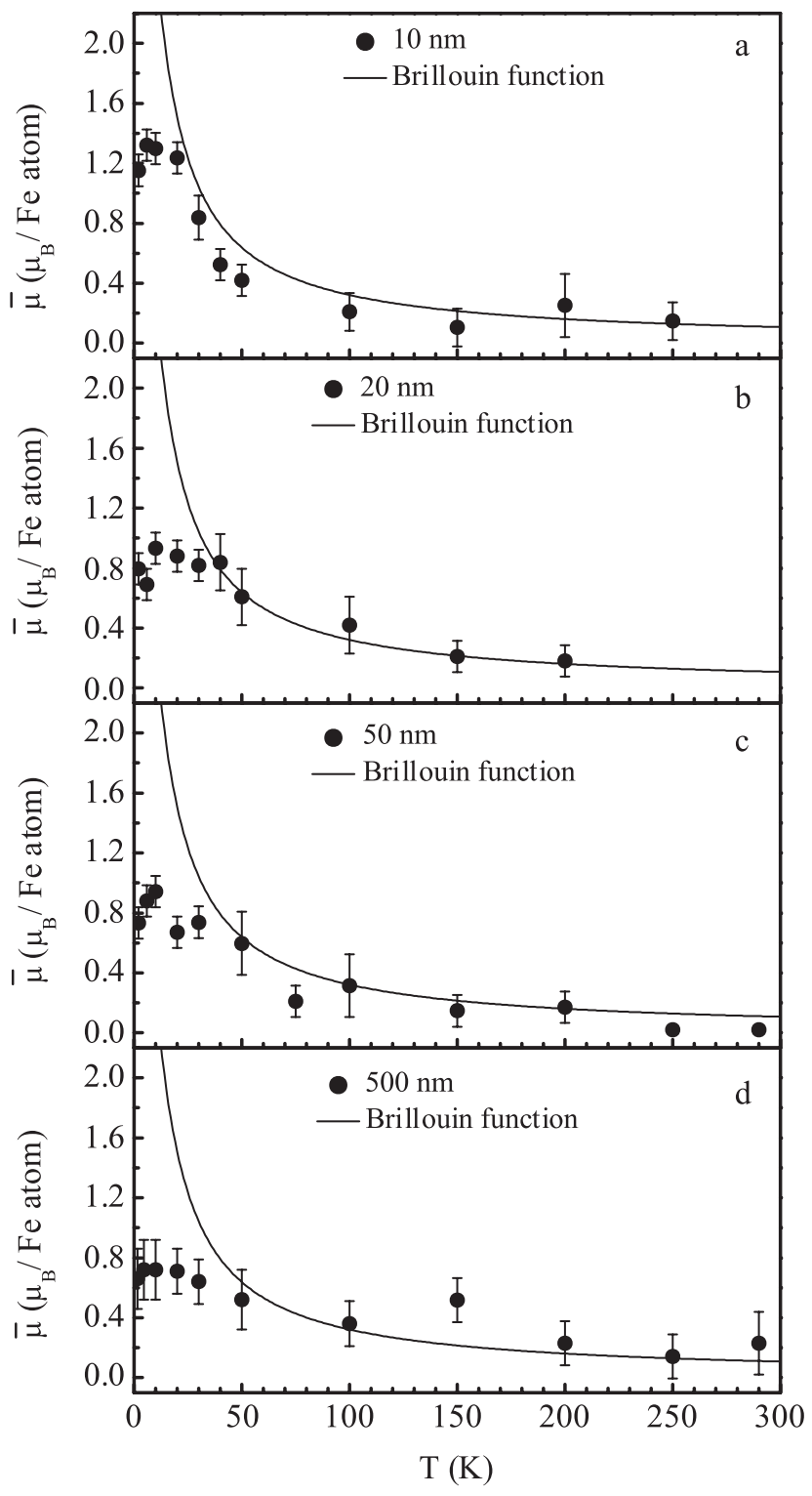

FIG. 3. Averaged magnetic moment per Fe atom versus temperature of thin $\mathrm{Au}_{97} \mathrm{Fe}_{3}$ films as derived from the fit to the PNR data (solid circles) compared to a Brillouin function with $J=2$ for isolated $\mathrm{Fe}$ atoms (solid line).

magnetic fields, because the observed cusp which is taken as the spin-glass signature could not be observed in large magnetic fields.

For $t=10 \mathrm{~nm}$ the onset of the spin-glass order $T_{0}$ is found to be slightly reduced compared to the bulk AuFe value. At the same time the reduction of $\bar{\mu}$, which indicates the robustness of the spin-glass order against the external field, is considerably smaller than found in our measurements on the thicker films. We clearly observe a spin-glass behavior at $t=10 \mathrm{~nm}$, although less robust, in agreement with magnetic susceptibility measurements on CuMn and AgMn multilayers [32] and low-energy muon spin rotation measurements [33]. Our conclusions are in contrast to 
earlier anomalous Hall effect measurements on AuFe films with 1 at.\% Fe where a destruction of the spin-glass order was concluded from the vanishing of the cusp [3] for $t \leq$ $12 \mathrm{~nm}$. As the cusp for the anomalous Hall effect and susceptibility vanishes in large magnetic fields as well, our data prove that a vanishing of a cusp not necessarily means a destruction of the spin-glass order.

It is impossible from our data set to deduce any type of scaling behavior or to decide whether the increase of the spin-glass magnetization at $t=10 \mathrm{~nm}$ might be due to the theoretically predicted surface anisotropy $[12-16]$. The aim of the present study was to prove the feasibility of observing the transition of a single thin film into a spinglass state by measuring the magnetization during the cooldown in a large magnetic field. The large magnetic field was applied to reach equilibrium in a short time and measure the true spin-glass magnetization. The fact that the magnetic moment deviates from the Brillouin curve at low temperatures shows that the interaction energy leading to the frustration is larger than the Zeeman energy of the spins in the applied field of $6 \mathrm{~T}$.

In conclusion, we have performed PNR measurements on thin $\mathrm{Au}_{97} \mathrm{Fe}_{3}$ single films in a field of $6 \mathrm{~T}$ and successfully determined the magnetization in a temperature range from 295 down to $2 \mathrm{~K}$. The magnetization can be described with a Brillouin function from $295 \mathrm{~K}$ down to a temperature $T_{0}$, followed by a saturation value for $T<T_{0}$. The observed $T_{0}$ is about $50 \mathrm{~K}$ down to a film thickness of $t=$ $20 \mathrm{~nm}$ and decreases to $20 \mathrm{~K}$ at $t=10 \mathrm{~nm}$. The saturation value down to $t=20 \mathrm{~nm}$ is $0.9 \mu_{B}$ per Fe atom, whereas it increases to $1.3 \mu_{B}$ at $10 \mathrm{~nm}$. Our data clearly prove a phase transition to a spin-glass state in large magnetic fields down to $t=10 \mathrm{~nm}$ and a size-dependence of the magnetization of thin AuFe spin-glass films. Our data also show that we can clearly identify the frustrated spin-glass state in large magnetic fields where other methods requiring a cusp-type behavior fail because of a vanishing of a cusp.

We thank M.P. Singh and P. Fournier, physics department of the University of Sherbrooke, Canada, for the SQUID measurements on our $500 \mathrm{~nm}$ thick AuFe film.

*helmut.fritzsche@nrc.gc.ca

[1] K. Binder and A.P. Young, Rev. Mod. Phys. 58, 801 (1986).

[2] V. Cannella and J.A. Mydosh, Phys. Rev. B 6, 4220 (1972).

[3] H. Vloeberghs, J. Vranken, C. Van Haesendonck, and Y. Bruynseraede, Europhys. Lett. 12, 557 (1990).

[4] R. J. Borg, R. Booth, and C. E. Violet, Phys. Rev. Lett. 11, 464 (1963).
[5] R.C. Sundahl, T. Chen, J.M. Sivertsen, and Y. Sato, J. Appl. Phys. 37, 1024 (1966).

[6] J. A. Mydosh, P. J. Ford, M. P. Kawatra, and T. E. Whall, Phys. Rev. B 10, 2845 (1974).

[7] P. J. Ford and J. A. Mydosh, Phys. Rev. B 14, 2057 (1976).

[8] A. K. Nigam and A. K. Majumdar, Phys. Rev. B 27, 495 (1983).

[9] D. S. Fisher and D. A. Huse, Phys. Rev. B 36, 8937 (1987).

[10] G. G. Kenning, J. M. Slaughter, and J. A. Cowen, Phys. Rev. Lett. 59, 2596 (1987).

[11] G. G. Kenning, J. Bass, W. P. Pratt Jr., D. Leslie-Pelecky, L. Hoines, W. Leach, M. L. Wilson, R. Stubi, and J. A. Cowen, Phys. Rev. B 42, 2393 (1990).

[12] O. Ujsághy, A. Zawadowski, and B. L. Gyorffy, Phys. Rev. Lett. 76, 2378 (1996).

[13] O. Ujsághy and A. Zawadowski, Phys. Rev. B 57, 11598 (1998).

[14] O. Ujsághy, L. Szunyogh, and A. Zawadowski, Phys. Rev. B 75, 064425 (2007).

[15] E. Seynaeve, K. Temst, F. G. Aliev, C. Van Haesendonck, V. N. Gladilin, V. M. Fomin, and J. T. Devreese, Phys. Rev. Lett. 85, 2593 (2000).

[16] V. N. Gladilin, V. M. Fomin, and J. T. Devreese, Physica (Amsterdam) 294-295B, 302 (2001).

[17] private communications with M. P. Singh and P. Fournier, physics department, University of Sherbrooke, Canada.

[18] J. Swerts, K. Temst, C. Van Haesendonck, H. Fritzsche, V. N. Gladilin, V. M. Fomin, and J. T. Devreese, Europhys. Lett. 68, 282 (2004).

[19] H. Fritzsche, M. Saoudi, K. Temst, and C. Van Haesendonck, Physica (Amsterdam) 397B, 47 (2007).

[20] C. N. Guy, J. Phys. F 5, L242 (1975).

[21] R. V. Chamberlin, M. Hardiman, L. A. Turkevich, and R. Orbach, Phys. Rev. B 25, 6720 (1982).

[22] L. E. Wenger and J. A. Mydosh, J. Appl. Phys. 55, 1717 (1984), and references therein.

[23] T. Duffield and C. N. Guy, J. Phys. F 15, L17 (1985).

[24] J. Bergsma and C. van Dijk, Nucl. Instrum. Methods 51, 121 (1967).

[25] H. Fritzsche, Rev. Sci. Instrum. 76, 115104 (2005).

[26] G. P. Felcher, R. O. Hilleke, R. K. Crawford, J. Haumann, R. Kleb, and G. Ostrowski, Rev. Sci. Instrum. 58, 609 (1987).

[27] S. J. Blundell and J. A. C. Bland, Phys. Rev. B 46, 3391 (1992).

[28] F. Radu, V. Leiner, M. Wolff, V. K. Ignatovich, and H. Zabel, Phys. Rev. B 71, 214423 (2005).

[29] L. G. Parratt, Phys. Rev. 95, 359 (1954).

[30] V.F. Sears, Neutron news 3, 26 (1992).

[31] J. J. Smit, G. J. Nieuwenhuys, and L. J. de Jongh, Solid State Commun. 32, 233 (1979).

[32] L. Hoines, R. Stubi, R. Loloee, J. A. Cowen, and J. Bass, Phys. Rev. Lett. 66, 1224 (1991).

[33] G. J. Nieuwenhuys et al., PSI reports 2003; http://num. web.psi.ch/reports/2003/LMU/lmu18-lem-SpinGlass.pdf. 